Perspective

\title{
Cell Biology
}

\section{Clogging information flow in ALS}

Joseph W. Paul III ${ }^{1,2}$ and Aaron D. Gitler ${ }^{1,3}$

1. Department of Genetics, Stanford University School of Medicine, Stanford, CA 94305

2. Department of Biology, East Carolina University, Greenville, NC 27858.

3. Correspondence should be addressed to: A.D.G.

\author{
Aaron D. Gitler \\ 300 Pasteur Drive \\ M322 Alway Building \\ Stanford, CA 94305 \\ agitler@stanford.edu
}

\section{NOTICE}

This is the author's version of the work. It is posted here by permission of the AAAS for personal use, not for redistribution. The definitive version was published in Science 5 September 2014: Volume 345, number 6201, pages 1118-1119, doi:10.1126/science.1259461.

Full text available with subscription or payment at:

http://www.sciencemag.org/content/345/6201/1118.long 
Amyotrophic lateral sclerosis (ALS), also known as Lou Gehrig's disease, is a devastating neurodegenerative disorder that causes a progressive loss of motor neurons, leading to paralysis and death typically within 2 to 5 years of onset. There are no cures and few treatments. ALS shares some genetic and pathological overlap with another neurodegenerative disease, frontotemporal dementia (FTD), which causes changes to personality and language. Mutations in the gene called chromosome 9 open reading frame $72(\mathrm{C} 9$ orf72) are the most common genetic cause of both ALS and FTD. On pages 1139 and 1192 of this issue, Kwon et al. (1) and Mizielinska et al. (2), respectively, describe how C9orf72 mutations might cause neurodegeneration.

Mutations in the $C 9$ orf72 gene were identified as the most common cause of ALS and FTD (3, 4). This discovery completely revolutionized research on both conditions, with intense efforts now focused on understanding the mechanisms by which C9orf72 mutations cause disease and developing therapeutic strategies to treat patients harboring these mutations. The mutational mechanism is a massive expansion of a hexanucleotide repeat, GGGGCC ( $\mathrm{G}$ is guanine and $\mathrm{C}$ is cytosine) located in a noncoding region of the $C 9 \operatorname{orf} 72$ gene. Normally, the number of repeat copies ranges from 2 to 23 , whereas hundreds or even thousands of copies constitute the disease-causing range. How do these extra GGGGCC repeats cause disease? There are three main hypotheses. One proposal is that expanse of repeats may interfere with the expression of $C 9$ orf 72 , thereby resulting in a loss of gene function that is detrimental (5). Another possibility is that the GGGGCC repeat transcript (as well as the antisense CCCCGG repeat transcript) may accumulate in RNA foci ( 6 ) and sequester RNA binding proteins, which could disrupt RNA metabolism. There is also the idea based on the perplexing finding that the long repeat is translated into protein in all six reading frames of the RNA (sense and antisense directions) in a manner that does not depend on the presence of the start codon ATG (A, adenine; T, thymine; G, guanine). This repeat-associated non-ATG translation (RAN translation) thus produces the dipeptide repeat proteins glycine-alanine (GA), glycine-proline (GP), proline-alanine (PA), glycine- 
arginine (GR), and proline-arginine (PR). These dipeptide repeat proteins are themselves aggregationprone and accumulate in affected brain regions (7). Of course, these three mechanisms are not mutually exclusive, but determining the contribution of each aberration will be critical for the development of effective therapeutic interventions.

To dissect potential contributions from the repeat RNA and those of the dipeptide translation products to disease, Mizielinska et al. generated repeat RNA constructs harboring interruptions that preclude their translation. The authors engineered a series of stop codons in both the sense and antisense RNAs every 12 GGGGCC repeats. This way, the RNA could be expressed but the dipeptide translation products would not. They validated that the pure repeats and interrupted repeats formed the G-quadruplex structures characteristic of GGGGCC repeats (5), indicating that the interruption strategy did not grossly affect the repeat structure. Both pure and interrupted constructs also formed RNA foci when expressed in cultured neuronal cells. The authors then generated transgenic fruit flies expressing either the pure or interrupted GGGGCC repeats. Remarkably, expression of pure repeats (able to form dipeptides) in the fly eye caused degeneration, whereas the interrupted constructs (producing RNA only) did not. Likewise, expressing pure repeats in the nervous system of adult flies led to toxicity and early lethality, whereas the interrupted repeats had no effect. Blocking translation by cycloheximide treatment of flies partially suppressed the phenotype, indicating that toxicity was attributable to translation. Thus, Mizielinska et al. demonstrate that the GGGGCC repeats can cause toxicity through the production of aberrant translation products and not from the RNA alone.

But which of the five possible dipeptides (GA, GP, PA, GR, PR) translated from the C9orf72 repeat drives neurodegeneration? Mizielinska et al. generated transgenic flies expressing each dipeptide independently, but not in the context of a repeat sequence - that is, rather than using GGGGCC to produce the dipeptides, because the genetic code is degenerate (more than one codon codes for the same amino acid), the authors could use different codons to make the dipeptides of 
interest (e.g., GR) without having a repetitive sequence. Expression of two of the dipeptides, GR and PR, was sufficient to cause toxicity and early lethality when directed to the eye or nervous system. The others were less toxic, but the GA dipeptide did show some toxicity later in life. These results focus attention on the arginine-containing dipeptides (GR and PR), and, to a lesser extent, GA, on driving neurodegeneration caused by C9orf72 mutations in FTD and ALS.

Kwon et al. also identify the arginine-containing C9orf72 dipeptides GR and PR as drivers of toxicity, but present a new hypothesis to explain how they might contribute to FTD and ALS pathogenesis. In a broader sense, their study connects two of probably the most exciting new concepts in ALS and FTD research: $C 9$ orf72 nucleotide repeat expansion and the remarkable property of some RNA binding proteins (e.g., FUS, EWSR1, TAF15, hnRNPA2) harboring low-complexity sequences (also known as prion-like domains) to form polymeric assemblies, which incorporate into hydrogels in vitro (8). Such assemblies are akin to RNA granules (e.g., P-bodies and stress granules) that play key regulatory roles in gene expression (9). Many of the RNA binding proteins that can associate with hydrogels have serine-arginine (SR) repeat domains. These SR domain-containing proteins are regulated by phosphorylation (on the serine residues of the SR domains) by kinases. Kwon et al. show that this phosphorylation can control the association of SR proteins with hydrogels made up of RNA binding proteins such as hnRNPA2. Phosphorylation releases the SR domain protein from the gel. Mutating most of the serines in the SR domain protein to glycine (e.g., to convert these domains to GR instead of SR) blocks the effects of the kinases, and the mutant SR domain proteins are no longer able to be released from the hydrogel.

Kwon et al. recognized that some of the dipeptide RAN translation products produced from the C9orf72 repeat transcripts resembled the mutated SR domains (e.g., GR and PR). The authors thus proposed an intriguing hypothesis: These RAN translation products associate with hydrogels (or similar types of assemblies in vivo, such as RNA-containing granules) but are impervious to the 
regulatory action of kinases, and consequently, might clog up the trafficking of SR domain-containing RNA binding proteins that are moving in and out of these granules to transfer information throughout the cell. This block in information flow (e.g., alterations in RNA processing) could lead to cellular dysfunction and might underpin neurodegeneration in ALS and FTD. Indeed, recombinant RAN translation products comprising either polymers of GR or PR bound hnRNPA2 hydrogels and were resistant to kinase treatment (just like the mutated SR domains). When Kwon et al. added these recombinant GR and PR proteins to cultured cells, they entered the cells, got into the nucleus, and then localized to the nucleolus, where they caused cell death (see the figure).

To determine why GR and PR proteins are toxic and to define the potential consequences of their accumulation in nucleoli, Kwon et al. added one of the dipeptides (PR) to cultured astrocytes (a key cell type relevant for ALS) and performed RNA sequencing to assess potential RNA processing alterations. They identified a number of splicing changes as well as changes in the amounts of different mRNAs and major impairments in synthesis of ribosomal RNA (rRNA). These results suggest that by binding to nucleoli and clogging multiple steps along the pathway of RNA biogenesis, dipeptide products of the $\mathrm{C} 9$ orf72 repeat expansion could cause disease. Kwon et al. hypothesize that the arginine-rich stretches of the GR and PR peptides make them rogue mimics of nuclear localization signals, which may facilitate their entering the nucleus and wreaking havoc on multiple steps of RNA biogenesis. The derangements in RNA processing pathways, perhaps initially mild but accumulating over decades, may eventually lead to neurodegenerative disease pathogenesis.

The studies of Mizielinska et al. and Kwon et al. strongly implicate dipeptide toxicity as a central component of neurodegeneration. A major challenge is to decipher the mechanism by which RAN translation products contribute to neurotoxicity, and to reexamine dipeptide pathology in human disease with a focus on distinguishing GR and PR from the other ones (e.g., GP, GA, PA). Another recent study by Su et al. (10) presents a potentially game-changing biomarker assay to detect and 
quantify one of the RAN translation products from patient cerebrospinal fluid. Assays for the abundance of the disease-associated dipeptides could be used in a clinical trial setting to monitor the efficacy of potential disease-modifying therapies that target the repeat RNA expansion. These approaches could include small molecules, as shown by Su et al. (10), and antisense oligonucleotides $(6,11,12)$. More focus on understanding the molecular mechanisms of RAN translation could reveal new and unexpected therapeutic strategies. Perhaps there are ways to specifically prevent the production of the more toxic dipeptides in exchange for expressing more of some of the benign ones. Unbiased screens using model organisms (yeast, flies, worms, etc.) to identify suppressors of dipeptide toxicity, combined with validation in human patient samples and cell lines, may also reveal mechanisms underpinning dipeptide toxicity and may suggest approaches to mitigate this toxicity.

\section{References}

1. Kwon, I., Xiang, S., Kato, M., Wu, L., Theodoropoulos, P., Wang, T., et al. (2014). Polydipeptides encoded by the C9orf72 repeats bind nucleoli, impede RNA biogenesis, and kill cells. Science, 345(6201), 1139-1145. doi:10.1126/science.12549172.

2. Mizielinska, S., Gronke, S., Niccoli, T., Ridler, C. E., Clayton, E. L., Devoy, A., et al. (2014). C9orf72 repeat expansions cause neurodegeneration in Drosophila through arginine-rich proteins. Science, 345(6201), 1192-1194. doi:10.1126/science.1256800

3. M. DeJesus-Hernandez et al., Expanded GGGGCC hexanucleotide repeat in noncoding region of C9ORF72 causes chromosome 9p-linked FTD and ALS. Neuron 72, 245 (2011).

4. A. E. Renton et al., A hexanucleotide repeat expansion in C9ORF72 is the cause of chromosome 9p21-linked ALS-FTD. Neuron 72, 257 (2011).

5. A. R. Haeusler et al., C9orf72 nucleotide repeat structures initiate molecular cascades of disease. Nature 507, 195 (2014). 
6. C. J. Donnelly et al., RNA toxicity from the ALS/FTD C9ORF72 expansion is mitigated by antisense intervention. Neuron 80, 415 (2013).

7. S. C. Ling, M. Polymenidou, D. W. Cleveland, Converging mechanisms in ALS and FTD: disrupted RNA and protein homeostasis. Neuron 79, 416 (2013).

8. M. Kato et al., Cell-free Formation of RNA Granules: Low Complexity Sequence Domains Form Dynamic Fibers within Hydrogels. Cell 149, 753 (2012).

9. T. W. Han et al., Cell-free Formation of RNA Granules: Bound RNAs Identify Features and Components of Cellular Assemblies. Cell 149, 768 (2012).

10. Y. R. Li, O. D. King, J. Shorter, A. D. Gitler, Stress granules as crucibles of ALS pathogenesis. The Journal of cell biology 201, 361 (2013).

11. I. Kwon et al., Phosphorylation-regulated binding of RNA polymerase II to fibrous polymers of low-complexity domains. Cell 155, 1049 (2013).

12. Su, Z., Zhang, Y., Gendron, T. F., Bauer, P. O., Chew, J., Yang, W.-Y., et al. (2014). Discovery of a biomarker and lead small molecules to target r(GGGGCC)-associated defects in c9FTD/ALS. Neuron, 83(5), 1043-1050. doi:10.1016/j.neuron.2014.07.041 